\title{
聚乙烯树脂的制备及其在拆分 DL-氨基酸方面的应用*
}

\author{
直 金占华** 何两林 \\ (南开大学高分子研究所天津)
}

\section{关娄词䍜夫曼降解、氮基酸拆分、手性配体树脂}

本文报道了用霍夫曼降解法以交联聚丙稀酰胺为母体，制备聚乙烯胺树脂. 转化率可达 $83 \%$,对诸因素对转化率的影响进行了讨论. 以此树脂为骨架, 制备含 L-脯氨酸-Cu(Ii) 的手 性配体树脂, 并以此为柱色谱固定相, 将 DL-色氨酸完全拆分, D型先于 $L$ 型流出色谱柱.

\section{一、实验部分}

1. 试剂与仪器 丙烯酰胺: 分析纯 $\mathrm{N}^{\prime} \mathrm{N}-$ 亚甲基双丙烯酰胺: 化学纯; 碱性次氯酸 钠: 于冰浴中向氢氧化钠溶液通入氯气; 甲醇: 分析纯; XW-Il 紫外检测仪（北京新技术应 用研究所); XWD 1 型记录仪 (上海自动化仪表三厂).

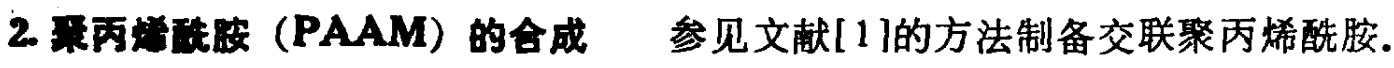

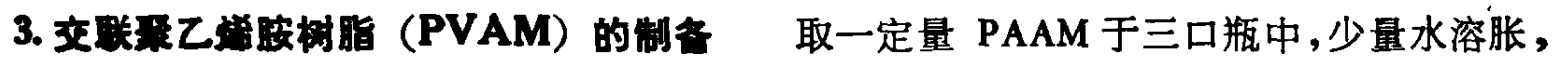
于冰盐浴中冷却,加人 $-5^{\circ} \mathrm{C}$ 左右的 $1.5 \mathrm{~mol} / \mathrm{L}$ 碱性次氯酸钠溶液, 达到 $-10^{\circ} \mathrm{C}$ 左右时, 加人 $10 \mathrm{~mol} / \mathrm{L}$ 氢氧化钠溶液,快速摚拌,于 $-10^{\circ} \mathrm{C} \sim-15^{\circ} \mathrm{C}$ 下反应 $2 \mathrm{~h}$, 继而于冰浴中反应 $15 \mathrm{~h}$. 反 应液倒人大量甲醇中, 得白色固体 (称中间产物). 将中间产物加水溶胀, 搅拌下滴加 $6 \mathrm{~mol} / \mathrm{L}$ 盐酸, 使 $\mathrm{pH}=2$, 抽潗, 以水、乙醇洗涤沉淀, 直到洗涤液以硝酸银检验时无白色沉淀生成为 止. $\mathrm{P}_{2} \mathrm{O}_{5}$ 真空干燥,得白色细粒状固体 (PVAM $\cdot \mathrm{HCl}$ ).

将 $\mathrm{PVAM} \cdot \mathrm{iICl}$ 于 $5 \%$ 氢氧化钠溶液中浸泡, 并转移到交换柱中继续以 $5 \%$ 氢氧化钠洗 染,水洗,倒出,抽干, $\mathrm{P}_{2} \mathrm{O}_{5}$ 真空干燥,得到白色细粒状固体 PVAM.

4. 转化率的河定在以盐酸水解中间产物时, 生成 $\mathrm{R}-\mathrm{NH}_{2} \cdot \mathrm{HCl}$, 将氯含量换算成胺 合量,进而可估算转化率. 同时将脱酸后的 PVAM 以酸碱滴定的方法测出胺含量, 直接估算 转化率,并将两种方法估算得到的转化率进行比较。氯含量的测定方法参见文献 [2].

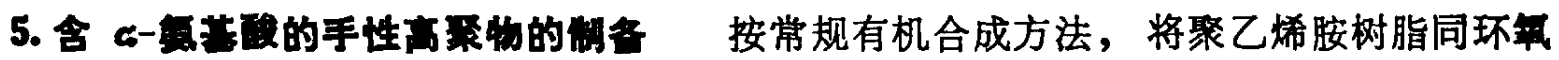
虫丙烷反应, 产物再与 $\alpha-$ 䀯虫酸氢氧化钠在水溶剂中反应, 得到含 $\alpha$-脯氮酸的手性配体树 脂, 将其以 $0.1 \mathrm{~mol} / \mathrm{L}$ 醋酸铜浸泡,充分络合 $\mathrm{Cu}$ (II).

\section{6. 柱色请法拆分 DL-色钅酸将含 L-脯氨酸的手性配体树脂装于 $0.7 \times 18 \mathrm{~cm}$ 玻琌}

本文 1988 年10月28日收到. 1989 年 1 月 22 日收到占、稆.

- 国家自然科学基金资助项目.

**北京化工学院. 


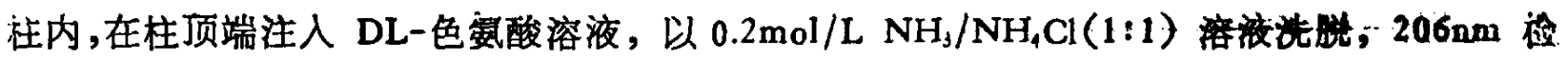
测。

\section{二、结果与讨论}

低分子酰胺在 $\mathrm{Cl}_{2} / \mathrm{NaOH}$ 存在下进行霍夫曼降解反应, 通常是在常温下进行的, 且当烷 基碳原子数超过 8 个时产率较低. 1976 年 Tanaka 等 ${ }^{[3,4]}$ 曾用霍夫曼降解法在低温下制得线 型聚乙烯胺. 我们以交联聚丙烯酰胺为母体, 在次氯酸钠和大过量氢氧化钠存在下, 低温下 进行霍夫曼降解, 产物经盐酸水解, 可得到聚乙烯胺树脂, 在显微镜下观察, 树脂表面光滑圆 亮,这说明此反应条件下并不破坏树脂的交联结构.

$$
\begin{gathered}
\mathrm{R}-\stackrel{\mathrm{O}}{\mathrm{C}}-\mathrm{NH}_{2} \stackrel{\mathrm{OC} 1-/ \mathrm{NaOH}}{\longrightarrow}[\mathrm{R}-\mathrm{N}=\mathrm{C}=\mathrm{O}\} \longrightarrow \mathrm{R}-\underset{\mathrm{NH}-\underset{\text { (中间产物) }}{\mathrm{C}}-\mathrm{ON2}}{\stackrel{\mathrm{O}}{\longrightarrow}} \\
\stackrel{\mathrm{H}+\mathrm{H}_{2} \mathrm{O}}{\longrightarrow} \mathrm{R}-\mathrm{NH}_{2}+\mathrm{CO}_{2} \uparrow
\end{gathered}
$$

交联 $10 \%$ 的聚丙烯酰胺若全部转化为聚乙烯胺, 则生成的 PVAM $\cdot \mathrm{HCl}$ 中氯含量约为 $41 \%$. 经实验, $1 \mathrm{~g}$ 交联 $10 \%$ 的 PAAM 经霍夫曼降解, 可得到 PVAM $\cdot \mathrm{HCl} 0.92 \mathrm{~g}$, 其氟含 量约为 $34 \%$, 若以氯含量估算转化率, 约为 $83 \%, \mathrm{~kg} \mathrm{PVAM} \cdot \mathrm{HCl}$ 脱盐酸, 可得到 PVAM $0.64 \mathrm{~g}$. 其胺含量为 $20 \%$,若以此估算转化率,约为 $80 \%$.

将交联 $10 \%$ 的 PAAM, 氯含量 $34 \%$ 的 PVAM $\cdot \mathrm{HCl}$ 及其脱盐酸后生成的 PVAM 样品 作元素分析,结果见表 1 .

将 PAAM、PVAM - $\mathrm{HCl}$ 及 PVAM 样品作红外分析, 对照结果表明, PVAM - $\mathrm{HCl}$ 样 品在 $2800 、 2000 \mathrm{~cm}^{-1}$ 附近的较宽吸收峰及 $1500 \mathrm{~cm}^{-1}$ 左右的吸收峰表明为 $-\mathrm{NH}_{\mathrm{s}}^{+}$基团, PVAM 中 $3300 、 3500 \mathrm{~cm}^{-1}$ 附近的两个吸收峰及 $1640 \mathrm{~cm}^{-1}$ 和 $770 \mathrm{~cm}^{-1}$ 处的吸收峰表明为 - $\mathrm{NH}_{2}$ 基团.

在进行霍夫曼降解时, 次氯酸钠的用量及碱与次氯酸钠的用量比 $R$ 对转化率的影响较火, 以 $r$ 表示次氯酸钠与酰胺基团的投料摩尔比, $R$ 表示碱浓度与次氯酸钠浓 度之比（ $R-$ $\left.\left[\mathrm{OH}^{-}\right] /\left[\mathrm{OCl}^{-}\right]\right)$. 转化率并非随次氯酸钠用量增大呈线性增长. 在 $r$ 为 $0.4-0.6$ 区间,基本 旺线性关系; 当 $r$ 超过 0.8 时, 次氯酸钠量对转化率影响不明显, 见图 1. $R$ 对转化率来说有一 最佳范围, 见图 2. $R$ 为 19 时转化率最高, 超过 19 后, 转化率明显下降, 这可能由于增大了碱 用量,相当于减少了次氯酸钠的浓度, 从而降低了它与底物的碰撞几率,减少了有效反应次数.

交联度在 5-35\% 的范围内变化对转化率没有明显的影响. 反应时间在 $15 \mathrm{~h}$ 内转化率明 显增长, $24 \mathrm{~h}$ 后基本趋于平衡。见图 3.

用于拆分 DL-氨基酸的配体交换树脂骨架种类见报道的仅有苯乙烯系、丙烯酰胺系和丙

表 1 元素分析结果

\begin{tabular}{c|c|c|c}
\hline & $\mathrm{C}(\%)$ & $\mathrm{H}(\%)$ & $\mathrm{N}(\%)$ \\
\hline PAAM & 44.36 & 7.04 & 15.73 \\
PVAM $\mathrm{HCl}$ & 30.32 & 7.72 & 16.25 \\
PVAM & 49.62 & 10.19 & 23.67 \\
\hline
\end{tabular}




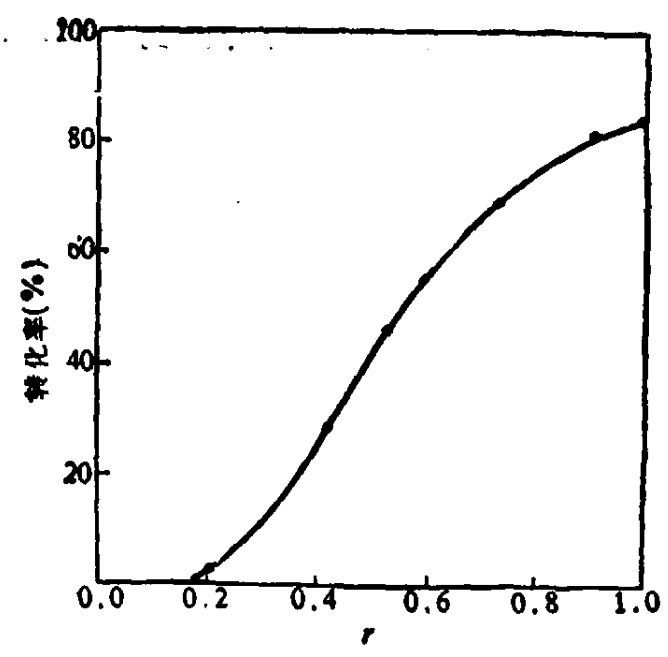

因 1 次督酸钠用量对转化率影响曲线
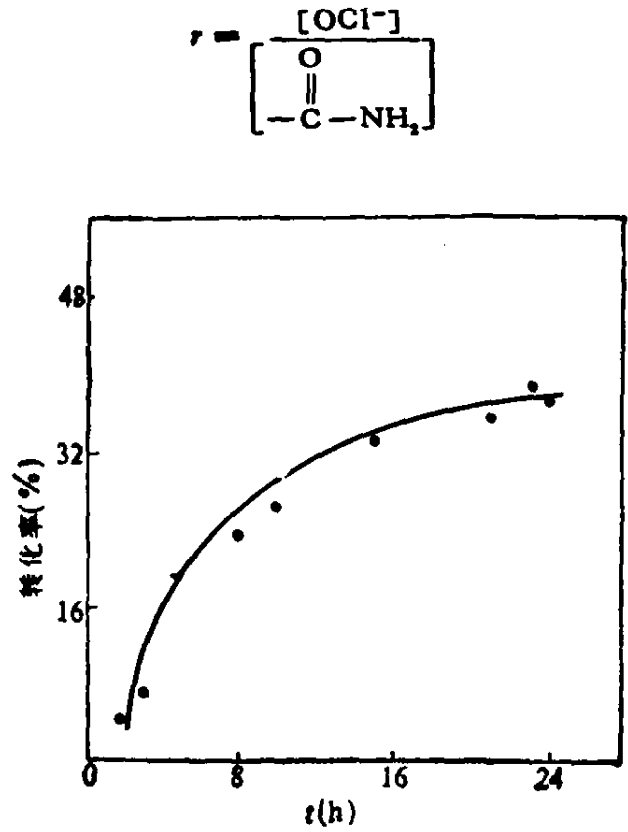

因 3 反应时间对转化率影响曲线

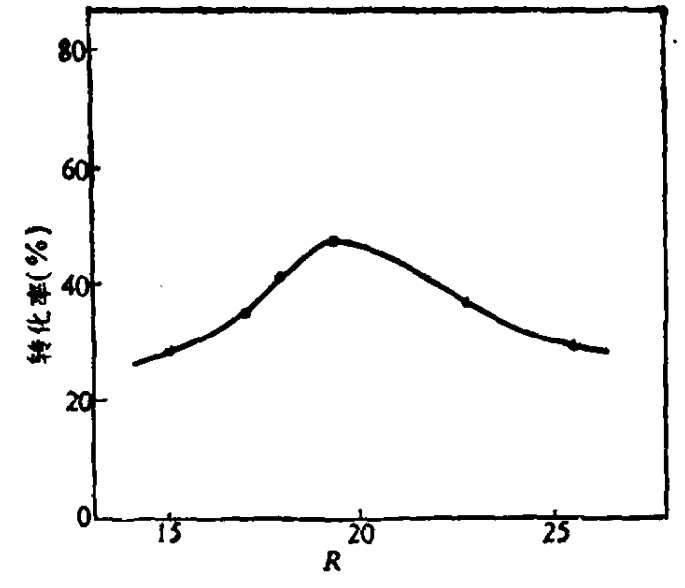

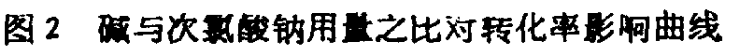
$r=0.46 ; R=\frac{\left[\mathrm{OH}^{-}\right]}{\left[\mathrm{OC}^{-}\right]}$

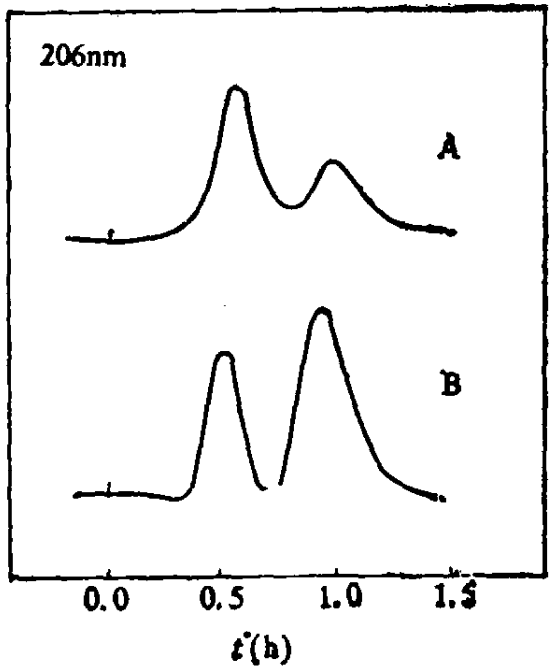

图 4 洗脱曲线

柱于: $0.7 \times 18 \mathrm{~cm}$, 流速: $10.6 \mathrm{ml} / \mathrm{h}$. 以 0.2 $\mathrm{mol} / \mathrm{L} \mathrm{NH} / \mathrm{NH}_{4} \mathrm{Cl}(1: 1)$ 洗脱. 曲线 $\mathrm{A}$ 进样为

DL-色氛酸;曲线 B 进样为 DL + I 色酸

烯酸醮系 ${ }^{[5-7]}$, 我们以聚乙烯胺树脂为骨架,合成了含 L-脯氨酸-Cu(II) 的配体交换树脂, 可 将 DL-色家酸完全拆分, 结果见图 4, 进样为 DL-色氨酸时, 得到两个分开的色谱峰, 说明 D 和 $L$ 型色氮酸可被分开; 当进样为 $D L+L$ 色氨酸时, 第二个峰明显突起, 说明第二个峰为 L-色氮酸，D型先流出色谱柱。

\section{$\Rightarrow$ 专 文 塥}

[1]铃木一,日本化学会志, 1975,1756 .

[2] R.A. 小定定分析, 1980,344.

[3] Tanaka, H., Polymer Letters Edition, 16(1978), 87.

[4] Tanaka, H. et al., Bulletin of the Chemical Society of Japan, 49(1976), 10: 2821.

[5] Davankov, U. A. et al., J. Chrom., 60(1971), 280.

[6] Yamskov, 1. A. et al., J. Chrom., 217(1981), 539.

[ 7 ] Leffebvre, B. et al., Isr J. Chem., 15(1977), 69. 